\title{
Bioindication of microcystins toxicity by germinating seeds
}

\author{
Z. Romanowska-Duda B. ${ }^{1}$, M. Grzesik ${ }^{2}$, J. T. Mankiewicz ${ }^{3}$ \\ \& M. Zalewski ${ }^{3}$ \\ ${ }^{1}$ Department of Ecophysiology and Plant Growth Development, \\ University of Lodz, Banacha 12/16, 90-237 Lodz, Poland \\ ${ }^{2}$ Department of Ornamental Nursery and Seed Science, \\ Research Institute of Pomology and Floriculture, Skierniewice, Poland \\ ${ }^{3}$ International Centre for Ecology, Polish Academy of Science, \\ Lodz, Poland
}

\begin{abstract}
The effects of microcystins, produced by Microcystis aeruginosa PCC 7820, on seed germination, seedling growth, activity of selected enzymes and membrane permeability were evaluated, in order to elaborate the biotests for natural solution toxicity indication in water reservoirs. Commercial seeds of four selected species were allowed to germinate at $20^{\circ} \mathrm{C}$ on filter paper moistened with: (i) water (control), (ii) three concentrations of microcystins cultures produced by Microcystis aeruginosa PCC 7820 or (iii) medium BG11 in which these cultures were conducted. To evaluate the sensitivity of these seeds to microcystins the number of the germinated seeds were counted and the length of roots and hypocotyls were measured 1, 2 and 3 days after sowing, while activity of RNase, phosphatase ( $\mathrm{pH} 6.0$ and $\mathrm{pH} 7.5)$, dehydrogenases and electrolyte leakage were measured after 8 and 24 hours of seed exposition in these compounds. The results showed that microcystins did not decrease significantly germination percentage and mean germination time of the tested seeds, except of $L$. sativa where the number of the germinated seeds was slightly reduced. A toxic effect of these solutions was exhibited by decreased root length in all germinated seeds, while hypocotyls length was not significantly affected by them. Microcystins also decreased the activity of the investigated enzymes and increased electrolyte leakage measured 8 and 24 hours after sowing. The research showed that growth of roots and activity of $\mathrm{RN}$-ase, phosphatase $(\mathrm{pH} 6.0$ and $\mathrm{pH} 7.5)$ or dehydrogenases, as well as electrolyte leakage from germinating seeds of selected species could be used for fast monitoring of the microcystin toxicity in water.

Keywords: germination, microcystins, monitoring, toxicity, seeds, Sinapis alba, Cardamine sp., Lactuca sativa, Sorghum saccharatum.
\end{abstract}




\section{Introduction}

Blue-green algal toxins present in water during algae blooming may cause serious diseases and fatal intoxication of humans as well as farm and wild animals [4]. Microcystins belong to these toxins and they are the most common hepatotoxins produced mainly by Cyanophyta from the genus Microcystis, as well as Anabaena. Their toxicity is much higher than that of strychnine and sodium cyanide [4]. It was showed that regular taking by human body of small doses of hepatotoxins from water reservoir, where algae are blooming, causes dyspepsia and may initiate liver cancer, bleeding, necrosis, as well as apoptosis in hepatocyte cells [5].

Increasing pollution of water environment with blue-green algal toxins has brought about the need to monitor their harmfulness, which would enable to undertake the actions focused on protection of conditions of human life. Most of the present methods of water pollution evaluation are based on chemical analyses of hazardous compounds. They are expensive, difficult to conduct and often indicate only some specific pollutants without information about their negative effect on organisms. Thus, in the preservation of human health and water quality, it is important to evaluate ecotoxicological effects of contaminated water to complement the techniques of analytical chemistry [12]. Resent reports show that bioindication has become the leading method in the world for monitoring pollution in water environment. This method utilises living organisms and their reactions as an indication of environmental pollution. In contrast to chemical tests bioindication assesses total toxicity of the environment and its summary effect. As a result of pollutants influence on the marker organisms variations from control organisms (untreated) are observed including morphological changes, retardation or induction of growth and mobility, occurrence of diseases and mortality rate.

The research has suggested that for these purposes animals, plants and germinated seeds that enable for rapid evaluation of pollution level of water can be employed $[8,9,10,13,14]$. Seed germination and root elongation technique seems to be an easy and inexpensive screening test and it can be easily monitored. Since the metabolism of seeds can be influenced by environmental conditions, phytotoxicity tests based on germination and root elongation to assess the potential contamination by metal ions were proposed recently $[1,8]$. Suggested plant species for phytotoxicity indication include cucumber, lettuce, radish, red clover, wheat $[3,6,18]$, oats, corn, cabbage, carrots, soybean, tomato, ryegrass, onions, beans [16], as well as rice, sorghum, mustard, rapeseed, turnip, vetch, fenugreek [2, 10, 17]. The used seeds can be exposed by floating in test solution, placed on filter paper soaked with the solution or sowed on sediments and tested soil, as well as deployed on a solid substrate and hydrated with reference water or test solution. They can be also exposed to aqueous extracts from soil or sediments [2].

Although the mentioned literature data indicate possibility to use seeds to monitor the toxicity of different compounds in water or soil, information concerning the exact sensitivity of seeds of particular species to stress caused by 
chosen pollutants is very scant. This applies also to the toxicity of blue-green algal toxins dissolved in water. Therefore, the aim of the presented research is to evaluate the effects of microcystins produced by Microcystis aeruginosa PCC 7820 on seed germination, selected physiological properties and growth of roots and hypocotyls of white mustard, cuckoo, lettuce and sorghum, in order to elaborate a fast and inexpensive biological test for the microcystin toxicity monitoring in water.

\section{Material and methods}

\subsection{Materials}

Commercial seeds of Sinapis alba (white mustard), Cardamine sp. (cuckoo), Lactuca sativa (lettuce), produced in the Central Poland and Sorghum saccharatum (sorghum), cultivated in South Italy, were used in experiment following the method described by Grzesik and Romanowska-Duda [8].

\subsection{Seed treatments}

For each experiment, $6 \mathrm{ml}$ of distilled water (control) or medium BG11 or three concentrations of microcystins cultures $(100000,200000,300000$ cells $/ \mathrm{ml}$, majority of MC-LR and MC-RR, identified with HPLC), produced by Microcystis aeruginosa PCC 7820 and cultivated in medium BG11, were added to a polystyrene Petri dishes $(60 \times 10 \mathrm{~mm})$ containing two Whatman No 1 filter paper discs. Seeds of the investigated species were placed in dishes containing the tested solutions. Seeds number in each dish or number of replicates for each tested sample depended on method of their evaluation. The plates with the moistened seeds were incubated at $20^{\circ} \mathrm{C}$ in the dark for $3-5$ days.

\subsection{Estimation of microcystins toxicity}

To assess the sensitivity of the germinating seeds to the tested medium and microcystins, the percentage of the germinated seeds, mean germination time (time to reach 50\% germination), length of roots and hypocotyls, were evaluated. Activity of $\mathrm{RN}$-ase, phosphatase $(\mathrm{pH} 6.0$ and $\mathrm{pH} 7.5)$, dehydrogenases and electrolyte leakage from seeds was observed additionally in Sinapis alba, Cardamine sp. and Lactuca sativa, which have been chosen to these measurements due to their fast germination, as compared to Sorghum saccharatum.

\subsubsection{Germination percentage and mean germination time}

To evaluate the germination percentage and mean germination time, the number of germinated seeds, exposed to microcystins, was counted every day on the base of three replicates of 50 seeds each. A seed was considered germinated, when ridicule emerged from the pericarp and was at least $1 \mathrm{~mm}$ long. Percentage of germinated seeds and mean germination time were calculated with Seed 
Calculator 3.0 programme developed by CPRO-DLO, Wageningen in the Netherlands.

\subsubsection{Length of roots and hypocotyls}

The length of roots and hypocotyls were measured 1, 2 and 3 days after sowing of 3 x 50 seeds to dishes containing microcystins cultures, by means of a ruler to the closest millimeter.

\subsubsection{RN-ase activity}

For RN-ase activity evaluation, 3 x $200 \mathrm{mg}$ of seeds, 8 and 24 hours after sowing on filter paper moistened with distilled water (control), medium BG11 and microcystins cultures, were ground in mortar and homogenised in $6 \mathrm{ml}$ of $0.1 \mathrm{M}$ Tris- $\mathrm{HCl}$ buffer ( $\mathrm{pH} 7.45$ ) containing $0.4 \mathrm{~g}$ of PVP (polynylpyrrolidone). The homogenate was centrifuged at $4500 \mathrm{~g}$ for 20 minutes. Supernatant was used for enzyme assays. All stages of the extraction procedure were carried out at $1-4^{\circ} \mathrm{C}$. Enzyme activity were defined as the amount of RN-ase, which at $260 \mathrm{~nm}$ released one absorbance unit of soluble nucleotide per gram of fresh matter, during 1 minute at $30^{\circ} \mathrm{C}$ [11].

\subsubsection{Phosphatase activity}

Acid and alkaline was evaluated using 3 x $200 \mathrm{mg}$ of seeds. Seeds, 8 and 24 hours after sowing on filter paper in dishes containing the tested solutions/cultures, were homogenized at $1-4^{\circ} \mathrm{C}$ in $6 \mathrm{ml}$ of $0.05 \mathrm{M}$ Tris- $\mathrm{HCl}$ buffer ( $\mathrm{pH} 7.45$ ) containing $0.4 \mathrm{~g}$ of PVP (polynylpyrrolidone). The homogenate was centrifuged at $4500 \mathrm{~g}$ for 20 minutes. Supernatant was used for enzyme assays. Constitutive phosphatase $(\mathrm{pH} 6.0$ and $\mathrm{pH} 7.5)$ activity was measured according to Knypl and Kabzińska [11]. One unit of activity was defined as the amount of enzyme, which hydrolyzed $1 \mu$ mol of substrate p-NPP during 1 minute at $30^{\circ} \mathrm{C}$.

\subsubsection{Dehydrogenase activity}

For total dehydrogenase activity evaluation, fifty seeds, in four replicates, were imbibed for 8 or 24 hours in Petri dishes on cotton layer, moistened with distilled water (control) or with the tested solutions/cultured. Then, samples of $0.2 \mathrm{~g}$ seeds were placed in Eppendorf tubes, grinded and incubated at $25^{\circ} \mathrm{C}$ for 24 hours in $1 \mathrm{ml} 0.1 \mathrm{M}$ sodium phosphate buffer, $\mathrm{pH} 7.2$ containing $0.7 \%(\mathrm{w} / \mathrm{v})$ of 2,3,5-triphenyl tetrazolium chloride. After that time samples were centrifuged $(5 \mathrm{~min}, 5000 \mathrm{x} \mathrm{g})$ and the pellet were extracted six times with $1 \mathrm{ml}$ acetone. The solution absorption was measured at $488 \mathrm{~mm}$. A standard curve was prepared from known concentration of formazan [7].

\subsubsection{Electrolyte leakage}

Electrolyte leakage was measured on the base of four replicates of 50 seeds each. Seeds, after 8 and 24 hours of exposition on filter paper to the tested compounds/cultures, were thoroughly dried between filter paper. Than, they were soaked in $3 \mathrm{ml}$ of water and incubated at $20^{\circ} \mathrm{C}$ for 2 and 4 hours and boiled for 10 minutes. Electrical conductivity (in $\mu \mathrm{S} \cdot 50$ seeds $^{-1} \cdot 3 \mathrm{ml} \mathrm{H}_{2} \mathrm{O}^{-1}$ ) was 
measured after 2 and 4 hour of soaking and after boiling. A microcomputer conductivity meter CC-551 (ELMETRON, Poland) was used to measure the electrical conductivities of the leachate from seeds. Results were expressed as the percentage of the total leakage from seeds.

For the obtained results, the standard errors $( \pm \mathrm{SE})$ for all parameters were calculated from values of the replicates.

\section{Results}

The obtained results showed that microcystins (mainly MC-LR and MC-RR), produced by Microcystis aeruginosa PCC 7820 and cultivated in medium BG11 were toxic to the germinating seeds and decreased some metabolic processes, dependently on plant species. The used concentrations of these compounds affected the germinating seeds similarly.
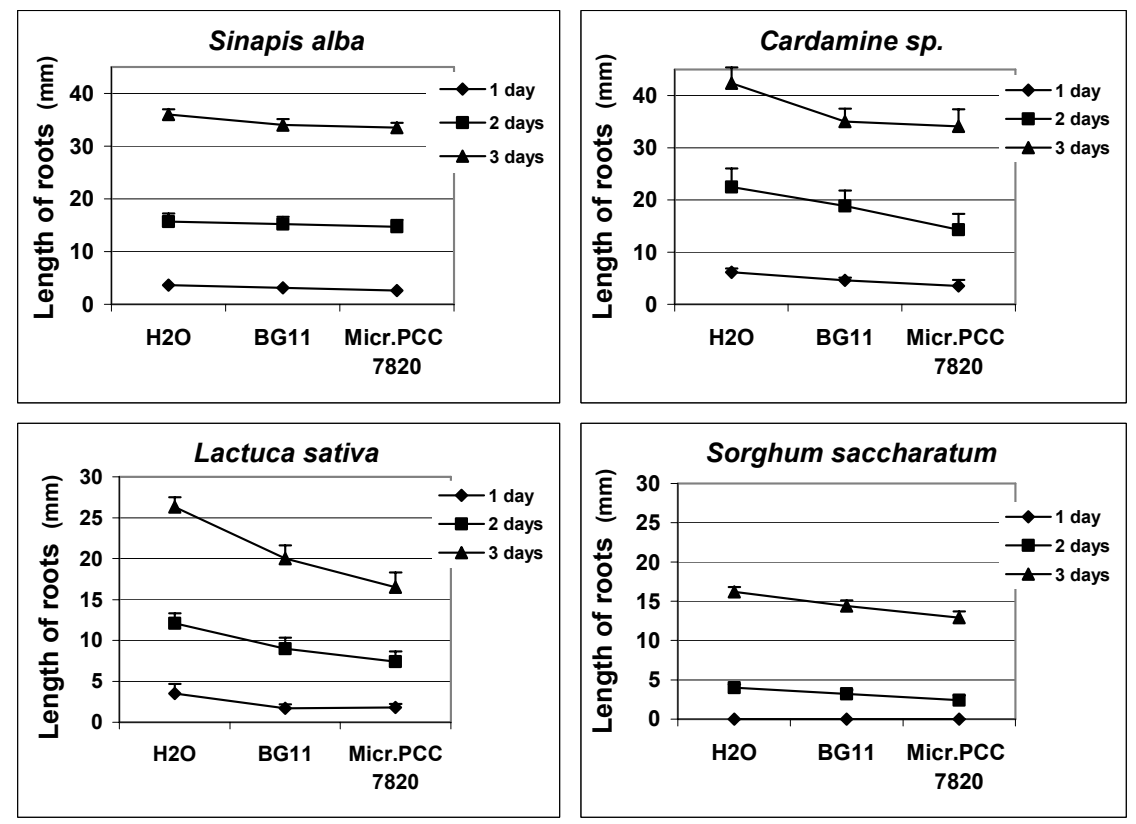

Figure 1: Length of 3-day-old roots grown at $20^{\circ} \mathrm{C}$, on filter paper moistened with distilled water (control), medium BG11 or microcystins produced by Microcystis aeruginosa PCC 7820 and cultivated in medium BG11. Error bars represent \pm SE.

The tested microcystins did not decrease significantly germination percentage and mean germination time (time to reach $50 \%$ of germination) of the tested seeds, which in normal conditions germinated in high degree and fast (all over 90\% and during one day, Sinapis alba, Cardamine sp.) or in lower percentage and slow (all 72\% and during 3-4 days, Sorghum saccharatum). In Lactuca 
sativa seeds, which germinated also over $90 \%$ and during one day, germination percentage was slightly reduced under exposition to medium BG11 and to higher extend due to the microcystins treatments.

Speed of germination, exhibited by mean time of germination of seeds of four tested species was not affected by microcystins and by medium BG11, in which Microcystis aeruginosa (producing these toxic compounds) were cultivated.
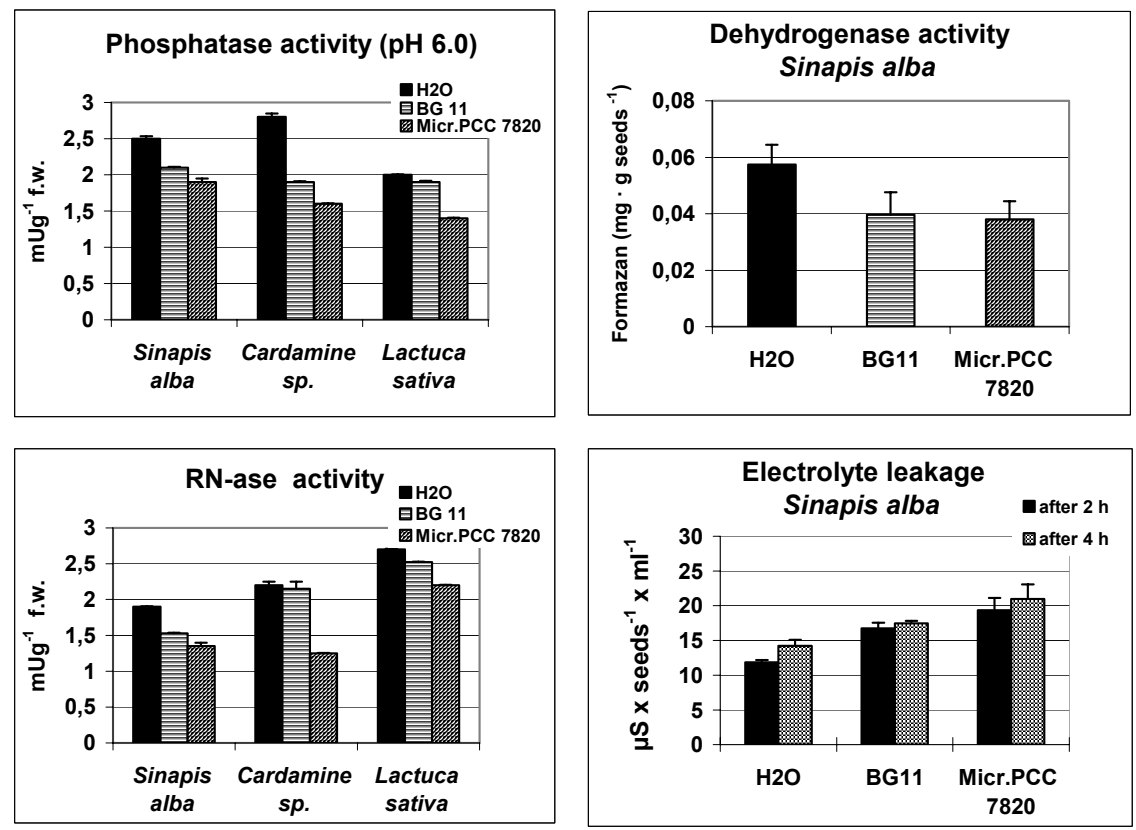

Figure 2: $\quad$ Activity of $\mathrm{RN}$-ase, acid ( $\mathrm{pH}$ 6.0) phosphatase or dehydrogenases and electrolyte leakage from embryos soaked for 2 and 4 hours in water, as affected by seed imbibition for 8 hours in distilled water (control), medium BG11 or cultures of microcystins produced by Microcystis aeruginosa PCC 7820 and cultivated in medium BG11. Error bars represent $\pm \mathrm{SE}$.

Toxic effect of the investigated microcystins to seeds of all four tested species was exhibited by decreased root length. The reductions in the root length, affected by microcystins, were mostly visible in Lactuca sativa and Cardamine $s p$. After 3 days of exposition to microcystins the length of roots in these both species was decreased by about $40 \%$ (from 27 to $17 \mathrm{~mm}$ ) and $20 \%$ (from 43 to $34 \mathrm{~mm}$ ), respectively, in comparison to control. Inhibiting influence of these compounds on root growth was also observed 2 and even 1 day after seed sowing. However, these reductions in the $2^{\text {nd }}$ and especially in the $1^{\text {st }}$ day were much less evident than after 3 days. Length of roots of Sinapis alba was reduced from $36 \mathrm{~mm}$ (in control) to $32 \mathrm{~mm}, 3$ days after seed sowing to microcystins 
cultures, while in Sorghum saccharatum this reduction was from 16 to $13 \mathrm{~mm}$. Reduced root length of the last both species, 2 and 1 day after sowing was no or barely noticeable (Fig. 1). The hypocotyl length was not affected significantly by the used solutions.

Cystins, produced by Microcystis aeruginosa PCC 7820, decreased activity of selected enzymes: RN-ase, acid ( $\mathrm{pH}$ 6.0) or alkaline ( $\mathrm{pH}$ 7.5) phosphatase, and dehydrogenases, in seeds exposed to these compounds/cultures for 8 and 24 hours. It was observed in Sinapis alba, Cardamine sp. and Lactuca sativa which have been chosen to these measurements due to their fast germination, as compared to Sorghum saccharatum. Activity of these enzymes was also reduced by medium BG11, in which Microcystis aeruginosa PCC 7820 (producing the evaluated microcystins) was cultivated. However, these reductions were always lower than in the case of microcystins and in some treatments they were very little (Fig. 2).

The evaluated microcystins evidently increased electrolyte leakage from seeds. The conductivity of water, in which seeds were soaked after previous exposition to microcystins for 8 hours, was much higher than in control.

Conductivity of water increased when time of soaking increased from 2 to 4 hours (Fig. 2). Similar dependencies were found when seeds were treated with microcystins for 24 hours.

\section{Discussion}

The presented research showed that toxicity of microcystins (mainly MC-LR and MC-RR), produced by Microcystis aeruginosa PCC 7820 cultivated in medium BG11 can be elevated by some physiological processes which premise or are subsequent to germination of Sinapis alba, Cardamine sp. Lactuca sativa and Sorghum saccharatum seeds.

The obtained results indicated that germination percentage and speed of germination of seeds of the tested species, expressed by mean germination time, can not be used for measuring of the toxic effects of microcystins in spite of the slightly decreased germination of Lactuca sativa seeds. This corresponds well with other our findings, when germination of seeds of the mentioned species was also not affected by salts of cadmium, copper, zinc and lead at concentration of $5-680 \mathrm{mg} \mathrm{L}^{-1}$ [8] and with the ascertainment of Kapustka and Reporter [10] that seed germination is rather insensitive to many toxic substances. Data concerning microcystins toxicity measurement with germinating seed it is hard to find in spite of several literature information in the toxicology field [9, 10, 13-15].

Toxic effects of the investigated microcystins to seeds of the four tested species can be elevated by decreased root length, which is very easy to measure and it is recommended for measurement of toxicity of some heavy metals $[1,8]$. The research shows that for the microcistins toxicity measurements the germinating seeds of Lactuca sativa and Cardamine sp. are more useful, due to their fast germination in normal conditions and higher sensitivity to microcystins, than Sinapis alba and Sorghum saccharatum. Because of uniform and rapid germination of Lactuca sativa and Cardamine sp., in just one day, root 
elongation values show excellent repeatability and for majority of samples significant differences between repetitions did not exceed a few millimeters. The length of roots exposed to microcystins was significantly decreased 3 days after seed sowing, although the first symptoms of inhibitions of the root growth can be observed 1 and 2 days after sowing. The sensitivity of the elongated roots of Lactuca sativa to some toxic compounds was also reported by Kapustka and Reporter [10].

Toxic effects of microcystis can also be monitored by measurements of activity of $\mathrm{RN}$-ase, acid ( $\mathrm{pH}$ 6.0) or alkaline ( $\mathrm{pH}$ 7.5) phosphatase, dehydrogenases and by increased electrolyte leakage from seeds of these both species tested. These measurements show the toxic effects after 8 hours of seed exposition to microcystins. This indicates that using seeds of Lactuca sativa and Cardamine sp. it is possible to elevate the toxicity of microcystins during 8 hours and these observations can be confirmed by the root length measurements after 1, 2 and 3 days of exposition to toxins. The possibility to use the activity of some enzymes for phytotoxicity assessment, such as activity of peroxidase and some isoenzymes was indicated also by some authors cited by Kapustka and Reporter [10]. Seeds of Sinapis alba seem to be a little less useful in the microcystins toxicity evaluation because the growing roots are less sensitive to toxic effects of these cultures. However, these seeds germinate in height percentage during one day. Sorghum saccharatum seeds germinate much slower than the mentioned three species, up to 3-4 days, and thus the results of measurements are delayed, when compared to Lactuca sativa and Cardamine sp. Similarly, hypocotyls length measurement seems to be less useful for testing of the microcystins toxicity than root length evaluation, because of their late growth. Toxic effects of microcystins produced by Microcystis aeruginosa PCC 7820 can be also indicated by Spirodela oligorrhiza, as it was found by Romanowska-Duda et al. [14] and Romanowska-Duda [15].

The presented research indicates that fast biomonitoring of toxic pollution in water by microcystins produced by Microcystis aeruginosa PCC 7820 can be conducted first of all by the following measurements in germinating seeds of Cardamine sp. and Lactuca sativa:

-length of roots, measured 3 days after seed sowing on filter paper moistened with the tested microcystins cultures

-electrolyte leakage in seeds treated with microcystins for 8 or 24 hours

-activity of enzymes: RN-ase, phosphatase ( $\mathrm{pH} 6.0$ and $\mathrm{pH} 7.5$ ) or dehydrogenases in seeds exposed to microcystins for 8 or 24 hours.

Lactuca sativa and Cardamine sp. seeds are cheap and easy to use for rapid evaluation of toxicity and thus they can be useful to monitor water pollution with the mentioned microcystins.

\section{Acknowledgement}

This study was supported by grant from the State Committee for Scientific Research, 3 PO4G 05925 and 2 PO4F 04427. 


\section{References}

[1] Barbero, P., Beltrami M., Baudo R. \& Rossi D., Assessment of Lake Orta sediments phytotoxity after the liming treatment. J. Limnol, 60(2), pp. 269-276, 2001.

[2] Beltrami, M., Rossi, D. \& Raudo, R., Phytotoxity assessment of Lake Orta sediments. Aquatic Ecosystem Health and Management. 2 pp. 391-401, 1999.

[3] Brusick, D.J., \& Young, R.R., IERL-RTP procedure manual: level I, environment assessment biological tests. US Environmental protection Agency, Washington, D.C.: EPA-600/8, pp. 81-02, 1981.

[4] Carmichael, W.W., Toxic microcystins and the environment. Toxic microcystins, ed M.F. Watanabe: CRS Pres pp. 1-10, 1996.

[5] Falconer, J.R., An overview of problems caused by toxic blue-green algae (Cyanobacteria) in drinking and recreational water. Environ. Toxicol. 14, pp. 5-12, 1999.

[6] Fletcher, J.S., Muhitch, M.J., Vann, D.R., McFarlane, J.C. \& Benenati, F.E., PHYTOTOX database evaluation of surrogate plant species recommended by the U.S. Environmental Protection Agency and the Organization for Economic Cooperation and Development. Environ Toxicol Chem. 4, pp. 523-532, 1985.

[7] Górnik, K. \& Grzesik, M., Effect of Asahi SL on China aster 'Aleksandra' seed yield, germination and some metabolic events. Acta Physiologiae Plantarum 24(4), pp. 379-383, 2002.

[8] Grzesik, M. \& Romanowska-Duda, Z., Effects of heavy metal salts on seed germination and seedling growth of white mustard and cuckoo flower (pol). X Ogólnopolski Zjazd Naukowy Hodowców Roślin Ogrodniczych. Skierniewice, 14-15.02.2005, pp. 256-262, 2005.

[9] Hardy, D., 10th International Symposium on Toxicity Assessment (ISTA 10). Quebec City, August 26 - 31: 2001, 2001.

[10] Kapustka, L.A. \& Reporter, M., Terrestrial Primary Producers. Handbook of Ecotoxicology. ed. P. Calow Ed. Oxford Blackwell Scientific Publications London Edinburgh Boston. I, pp. 278-299, 1994.

[11] Knypl, J.S. \& Kabzińska, E., Growth, phosphatase and ribonuclase activity in phosphate deficient Spirodela oligorrhiza cultures. Biochem Physiol Pflanzen 171, 279-287, 1977.

[12] Mendonca, E. \& Picado, A., Ecotoxicological monitoring of remediation in a coke oven soil. Environ Toxicol.,17 (1), pp. 74-9, 2002.

[13] Romanowska-Duda, Z. \& Tarczyńska, M., The influence of MicrocystinLR and hepatotoxic cyanobacterial extract on waterplant (Spirodela oligorrhiza). Environm. \& Toxicology. John Wiley and Sons, Inc., 17 (3), pp. 383-390, 2002.

[14] Romanowska-Duda, Z., Jurczak, T., Grzesik, M. \& Tarczyńska, M., Allelopatic relations between metabolities from Cyanobacteria against water plant (Spirodela oligorrhiza). VI International Conference on 
Toxic Cyanobacteria. Bergen, Norvay 21-27.06.2004. Abstracts, pp. 56, 2004.

[15] Romanowska-Duda, Z., Spirodela oligorrhiza (Kurz.) Hegelm. as bioindycator of toxicity of land water by hepatotoxins of cyanobacteria and heavy metals (pol). Habilitation thesis. University of Lodz, /in print/, 2005.

[16] US EPA., Ecological Effects Test Guidelines. OPPTS 850.4200. Seed germination/Root Elongation Toxicity Test. EPA 712-C-96-154, 1996.

[17] OECD, Terrestrial Plants: Growth test. OECD Guidelines for testing of chemicals. Paris, No. 208, 1984.

[18] Wang, W. \& Wiliams, J.M., Screening and biomonitoring of industrial effluents using phytotoxity tests. Environ. Toxicol Chem 7, pp. 645-652, 1988 . 